\title{
Primary oil processing optimization
}

\author{
Michael Livshits ${ }^{1, *}$, and Alexander Sizikov ${ }^{1}$ \\ ${ }^{1}$ Samara State Technical University, 443100 Samara, Russia
}

\begin{abstract}
The paper discusses oil distillation into target products with regard to the unclear separation effect. As the input data, the table of physical and chemical properties of narrow fractions is used. The problem is solved using the dynamic programming method. The methodology is realized in the authors' software product.
\end{abstract}

Primary oil processing takes place mostly in CDU (crude distillation units). There oil is separated in a number of target products: gasoline, kerosene, diesel, vacuum tower bottoms. Oil is characterized by a true boiling temperature (TBT) curve reflecting the relation between the temperature and distillation cuts' fractions. In practice, TBT is a compilation of narrow fractions with end points $\left[t_{i}, t_{i}+\Delta t_{i}\right], i=1,2, \ldots L$, where $L$ is the number of narrow fractions with $\Delta t=5 \div 10^{\circ} \mathrm{C}$.

The target product quality parameters depend on the target product temperature limits, i.e. the number of narrow fractions based on which separation is carried out $[1,2]$. The problem is reduced to establishing an option of separation at which the requirements to the target products' quality are met and the maximum of some criterion is achieved. It is also necessary to take into account that the current industrial units do not provide for an absolute clear separation of oil into fractions due to which temperature overlapping occurs (Fig.1).

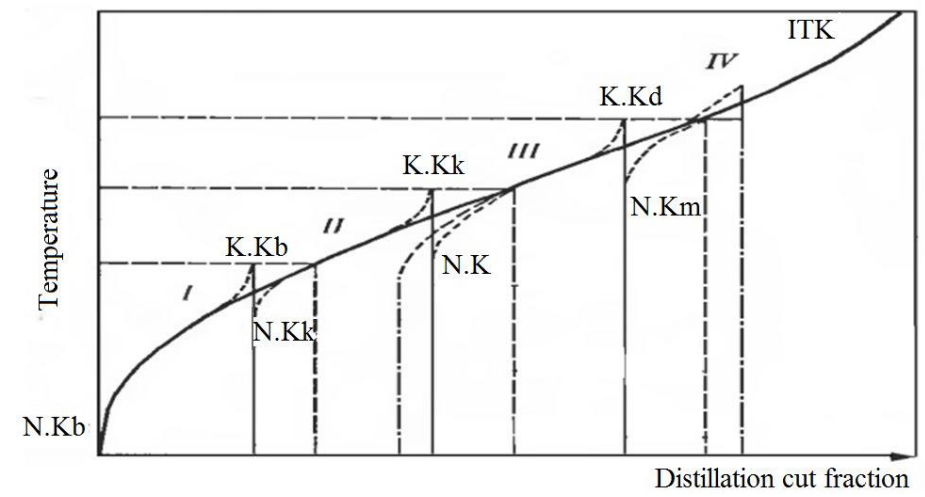

Fig. 1. Combined diagram of TBT curves of oil and product fractions.

Let's define the mathematical programming problem:

\footnotetext{
* Corresponding author: entcom@samgtu.ru
} 


$$
\max _{x}\left\{\sum_{n=0}^{N} f_{n}\left(x_{n}, x_{n+1}\right) \mid 0=x_{0} \leq x_{1} \leq \ldots \leq x_{N}=L\right\}
$$

Here $N$ is the number of target products; $x_{n}$ is the initial fraction of the $n$-th target product; $f_{n}\left(x_{n}, x_{n+1}\right)$ is vector criterion convolution characterizing the target product. The vector criterion convolution

$$
f_{n}\left(x_{n}, x_{n+1}\right)=\pi_{n} \widehat{g}_{n}\left(x_{n}, x_{n+1}\right)-\beta_{n} \psi_{n}\left(x_{n}, x_{n+1}\right)
$$

consists of two components. Component $\pi_{n} \widehat{g}_{n}\left(x_{n}, x_{n+1}\right)$ reflects the value of a respective target product; $\beta_{n} \psi_{n}\left(x_{n}, x_{n+1}\right)$ reflects the level of its compliance with the specification.

Here $\pi_{n}$ is the price or evaluation of the $n$-th product; $\beta_{n}$ is the convolution coefficient, with the help of which the correlation between criterion components is set; $\widehat{g}_{n}\left(x_{n}, x_{n+1}\right)=\sum_{i=x_{n}}^{x_{n+1}} g_{i}$ is the relative mass of the product in the oil mass; $g_{i}$ is the mass fraction of the $i$-th narrow fraction in oil.

To assure product quality parameters, a penalty component is introduced into (2)

$$
\psi_{n}\left(x_{n}, x_{n+1}\right)=\sum_{k=1}^{K} \xi_{n k} \delta_{n k}^{2}\left(x_{n}, x_{n+1}\right)
$$

As a weighted sum of squares of relative deviations:

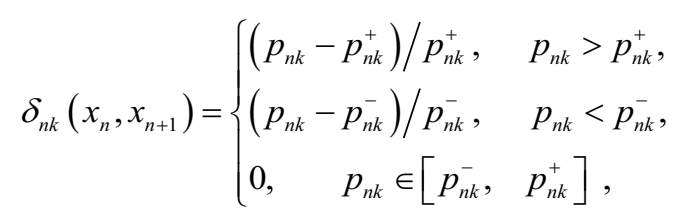

Here: $p_{n k}=p_{n k}\left(x_{n}, x_{n+1}\right)=I_{k}^{-1}\left(p_{n k}^{I}\left(x_{n}, x_{n+1}\right)\right)$ is the computed value of the parameter; $K$ is the number of quality parameters; $\xi_{n k}$ is the expert appraisal of the parameter priority; $p_{n k}^{-}, p_{n k}^{+}$are the lower and higher tolerable limits of the parameter; $p_{n k}^{I}\left(x_{n}, x_{n+1}\right)$ is the parameter index additively determined through indices of an appropriate parameter of the narrow fractions integrated by the product:

$$
p_{n k}^{I}\left(x_{n}, x_{n+1}\right)=\sum_{i=x_{n}}^{x_{n+1}} \rho_{i k}^{I} g_{i} \widehat{g}_{n}{ }^{1}
$$

where $\rho_{i k}^{I}=I_{k}\left(\rho_{i k}\right)$ is the index of parameter $\rho_{i k}$ of a narrow fraction; $I, I^{-1}$ are the direct and inverse functions of index transformations [2]

To account for the overlapping effect due to which fractions from neighboring cuts get into the product, it is suggested to proceed from the assumption that each product is formed from the whole range of narrow fractions taken with some weight coefficients $\mu_{i j}$ depending on the distance between fractions, $|i-j|$ : 


$$
\begin{gathered}
\widehat{g}_{n}=\sum_{i=x_{n}}^{x_{n+1}}\left(\sum_{j=1}^{L} \mu_{i j} g_{j}\right), \\
p_{n k}^{I}=\sum_{i=x_{n}}^{x_{n+1}}\left(\sum_{j=1}^{L} \mu_{i j} \rho_{j k}^{I} g_{j}\right) \cdot \widehat{g}_{n}^{-1}, k=1,2, \ldots K .
\end{gathered}
$$

In this instance, as a working hypothesis, it is naturally to assume that the weight coefficient decreases exponentially from $|i-j|$. Then, using normalization so that the sum of progression terms would be equal to one, we will get:

$$
\mu_{i j}=\left\{\begin{array}{c}
0, \quad|i-j|>m, \\
\frac{1-q}{2\left(1-q^{m}\right) q^{|i-j|}},|i-j| \leq m,
\end{array}\right.
$$

Here $q$ is the geometric ratio; $m$ is the number of progression terms equal to $d$ for all fractions except for extremes (the beginning and the end), for which

$$
m=\left\{\begin{array}{cl}
i, & i<d, j<i, \\
L-i, & i>L-d, j>i .
\end{array}\right.
$$

The values of parameters $q$ and $d$ are determined based on condition

$$
\min _{q, d} \sum_{n=1}^{N} \sum_{k=1}^{K}\left(\frac{p_{n k}-\hat{p}_{n k}}{P_{n k}}\right)^{2} .
$$

Problem (1) is solved by the dynamic programming method [3]. The process state before the $n$-th step means the initial fraction of the current target product, $S_{n-1}=x_{n-1}$; control is the number of narrow fractions included in the current product: $u_{n}=x_{n}-x_{n-1}$. The operator of transition into a new state is $S_{n}=x_{n-1}+u_{n}=x_{n}$. The step result functions should be understood as $f_{n}\left(S_{n-1}, u_{n}\right)=f_{n}\left(x_{n-1}, x_{n}\right)$. In this case, the recurrent Bellman equation will look as follows:

$$
F_{n-1}\left(x_{n-1}\right)=\max \left\{f_{n}\left(x_{n-1}, x_{n}\right)+F_{n}\left(x_{n}\right)\right\}, n=1,2, \ldots N,
$$

where $F_{n}$ is state function, $F_{n}\left(x_{n}\right)=0$.

The method of solving the postulated problem is as follows.

1. Within the range of tolerable variations of temperature limits, a net oriented graph so that the set of its paths would correspond to the set of tolerable solutions of the problem. Nodes are grouped by levels corresponding to process steps and form sets $X_{0}, X_{1}, \ldots$, $X_{N}$, wherein sets $X_{0}$ and $X_{N}$ contain one node. The distances between neighboring nodes are calculated by formula (2).

2. The computation procedure is executed from the end to the beginning: $n=N-1, N-2, \ldots, 0$. At each step, for all $x_{n} \in X_{n}$ problem (11) is solved, control $\widehat{u}_{n}\left(x_{n}\right)$ and potential $F_{n}\left(x_{n}\right)$ are established. 
3. By determining the every next optimal point through the previous one in chain: $\widehat{x}_{1}=\widehat{u}_{0}(0) \rightarrow \widehat{x}_{2}=\widehat{u}_{1}\left(\widehat{x}_{1}\right) \rightarrow \ldots \rightarrow \widehat{x}_{N}=\widehat{u}_{N-1}\left(\widehat{x}_{N-1}\right)$, we receive solution: $\widehat{x}_{1}, \widehat{x}_{2}, \ldots \widehat{x}_{N}$.

4. We determine values of separation temperature points and coefficients of selections of target products:

$$
T_{n}=t_{\widehat{x}_{n}}, \quad a_{n}=\widehat{g}_{n}=\sum_{i=x_{n}}^{\hat{x}_{n+1}}\left(\sum_{j=1}^{L} \mu_{i j} g_{j}\right), n=1,2, \ldots N .
$$

The methodology described is realized in the authors' software product [4].The latter can be used both on its own and within a higher level system, as part of a software for technical and economic modeling of a refinery [5].

\section{References}

1. M.Yu. Livshits, A.P. Sizikov, EPJ Web Conf. 110, 01035 (2016)

2. E.E. Dudnikov, Yu.M. Tsodikov, Typical Problems of Continuous Production Management (Energy, Moscow, 1979)

3. R. Bellman. Applied Dynamic Programming (Nauka, Moscow, 1965)

4. A.P. Sizikov. The System of Modelling and Optimization of Primary Oil Processing (SMOPOR). A.P. Sizikov; applicant and right holder of Samaraneftekhimproekt JSC RU).State Registration Certificate for Computer Program No. 2011618611 (15.09.2011)

5. A.P. Sizikov, Bulletin of the Samara State Technical University 7, 38 (2010) 